\title{
Aminoguanidine does not inhibit the initial phase of experimental diabetic retinopathy in rats
}

\author{
H.-P. Hammes ${ }^{1}$, S. Syed Ali ${ }^{2}$, M. Uhlmann ${ }^{1}$, A. Weiss ${ }^{1}$, K. Federlin ${ }^{1}$, K. Geisen ${ }^{3}$, M. Brownlee ${ }^{4}$ \\ ${ }^{1}$ Third Medical Department, Justus-Liebig-University of Giessen, Germany \\ ${ }^{2}$ Institute of Anatomy, Justus-Liebig-University of Giessen, Germany \\ ${ }^{3}$ Hoechst Pharma Research, Frankfurt/Hoechst, Germany \\ ${ }^{4}$ Diabetes Research Center and Departments of Medicine and Pathology, Albert Einstein College of Medicine, Bronx, New York, \\ USA
}

Summary We have previously shown that long-term administration of aminoguanidine, an inhibitor of advanced glycosylation product formation, reduces the extent of experimental diabetic retinopathy in the rat by $85 \%$. In order to determine whether the residual retinopathy that developed despite aminoguanidine was attributable to advanced glycation endproduct formation, a time-course study was performed in three different groups of male Wistar rats: non-diabetic controls (NC), streptozotocin-diabetic controls (DC) and streptozotocin-diabetic rats treated with aminoguanidine HCL, $50 \mathrm{mg} / 100 \mathrm{ml}$ drinking water (D-AG). Eyes were obtained at 24, 32, 44 and 56 weeks of diabetes/treatment duration and morphologic evaluation was done on retinal digest preparations. At 56 weeks, retinal basement membrane thickness was additionally measured. After 24 weeks of diabetes, the number of acellular capillaries was significantly elevated in DC $\left(44.6 \pm 5.7 / \mathrm{mm}^{2}\right.$ of retinal area, NC $19.6 \pm 4.9 ; p<0.001)$ and increased continuously over time (DC 56 weeks $87.4 \pm 15.1$; $p<0.001$ vs DC 24 weeks). In contrast, acellular capillaries in D-AG increased over the first 24 weeks and then remained constant for the rest of the study (DAG 24 weeks $35.7 \pm 5.18 ; p<0.01$ vs NC 24 weeks and NS vs DC 24 weeks; D-AG 56 weeks $42.0 \pm 6.20$; $p$ NS vs D-AG 24 weeks). Diabetes-associated pericyte loss (DC 24 weeks $2310 \pm 170 / \mathrm{mm}^{2}$ of capillary area; NC 24 weeks $3120 \pm 190 ; p<0.001 ; \quad D C$ 56 weeks $1570 \pm 230 ; \quad$ NC 56 weeks $2960 \pm 50$; $p<0.001$ ) was significantly prevented by aminoguanidine after diabetic-like changes over the initial 24 weeks (D-AG 24 weeks $2450 \pm 75 ; p$ NS vs DC 24 weeks; D-AG 56 weeks $2350 \pm 90 ; p<0.001$ vs DC 56 weeks). At 56 weeks, aminoguanidine treatment was associated with a $67.4 \%$ reduction in retinal basement membrane thickening. This time-course study demonstrates that aminoguanidine prevents the progression of experimental diabetic retinopathy, and suggests that non AG-inhibitable mechanisms are involved in the initial phase of diabetic retinopathy. [Diabetologia (1995) 38: 269-273]

Key words Diabetic retinopathy, rat model, aminoguanidine, glycation, retinal basement membrane.
Diabetic retinopathy is the most common microvascular disease affecting virtually every patient who has insulin-dependent mellitus diabetes for 15 years

Received: 16 June 1994 and in revised form: 29 August 1994

Corresponding author: Dr. H.-P. Hammes, Third Medical Department, Justus-Liebig-University, Rodthohl, D-35385 Giessen, Germany

Abbreviations: NC, Non-diabetic controls; DC, diabetic controls; D-AG, diabetic rats treated with aminoguanidine; $A G$, aminoguanidine; AGE, advanced glycation end products; STZ, streptozotocin; PAS, periodic acid Schiff. or longer [1]. Although the final clinical outcome greatly varies due to factors such as genetic determinants of individual susceptibility, hypertension and lipid status, the severity of retinopathy is associated with the degree of chronic hyperglycaemia [2-4]. From experimental as well as from clinical studies, there is evidence that the glycaemic history of the pre-complication period preceding overt diabetic retinopathy is a major determinant of the clinical outcome, indicating the relevance of hyperglycaemic memory in the pathogenesis of this complication [47]. The biochemical basis of this phenomenon may in- 
volve the formation and accumulation of advanced glycation products on long-lived extra- and intracellular molecules, leading to matrix crosslinks, altered cell-matrix interaction and DNA damage [8]. Thus, basement membrane thickening, a generally observed feature of diabetic vasculature, could result from vascular wall advanced glycation end-product (AGE) accumulation.

In support of the possible role of AGEs in the pathogenesis of diabetic retinopathy, it has been shown that long-term administration of aminoguanidine, an inhibitor of $\mathrm{AGE}$ formation, for 75 weeks reduces the extent of experimental diabetic retinopathy in the rat by $85 \%$. This effect is preceded by the inhibition of autofluorescence after 24 weeks [9].

In order to determine whether the residual retinopathy was due to AGE-formation, a time course study was performed in three different groups of rats investigating the effect of aminoguanidine treatment on histological parameters of retinopathy including basement membrane thickening in the streptozotocin (STZ)-diabetic rat model.

\section{Materials and methods}

Animals. Male Wistar rats (strain SPF 71, Hoechst-AG, Frankfurt-Hoechst, Germany) weighing $200 \pm 2.6 \mathrm{~g}$ at the outset were used in this experiment. Diabetes was induced by iv injection of streptozotocin (STZ, $60 \mathrm{mg} / \mathrm{kg}$ body weight; Sigma, München, Germany). Rats were fed a regular laboratory rodent chow ad libitum and had free access to drinking water. Animals with blood glucose exceeding $15 \mathrm{mmol} / 1$ at 3 weeks after STZ-injection received either no treatment or $50 \mathrm{mg} /$ $100 \mathrm{ml}$ drinking water aminoguandine-HCL (Riedel-de Haen, Hannover, Germany). Water consumption was identical in the treated and the untreated diabetic groups.

Blood glucose, body weight and water consumption were checked biweekly and glycated haemoglobin (Glyc Affin $\mathrm{GHb}$, Isolab, DRG, Marburg, Germany) was measured at the time of killing.

Animals were killed at 24 weeks (non-diabetic rats (NC) $n=6$, diabetic (DC) $n=5$, diabetic treated (D-AG) $n=5$ ), 32 weeks (NC $n=5, \mathrm{DC} n=5, \mathrm{D}-\mathrm{AG} n=6$ ), 44 (NC $n=6$, DC $n=6, \mathrm{D}-\mathrm{AG} n=8$ ) and 56 weeks of diabetes (NC $n=5$, $\mathrm{DC} n=8, \mathrm{D}-\mathrm{AG} n=6$ ).

Retinal digest preparations. Retinae were obtained at the time points indicated above after enucleation of the eyes from the animals under deep anaesthesia and immediately fixed in $4 \%$ buffered formalin.

Retinal vascular preparations were performed using a pepsin-trypsin digestion technique as previously described $[9,10]$. Briefly, a combined pepsin (5\% pepsin in $0.2 \%$ hydrochloric acid for $1.5 \mathrm{~h}$ ) trypsin ( $2.5 \%$ in $0.2 \mathrm{~mol} / 1$ Tris for $15-30 \mathrm{~min}) \mathrm{di}-$ gestion was used to isolate the retinal vasculature and the specimen were stained with periodic acid Schiff (PAS).

Acellular capillaries were quantitated by a modification of the method of Engerman and Kern [6]. Using a grid of 100 fields, 10 microscopic fields covering a total area of $6.76 \mathrm{~mm}^{2}$ of retinal area were scored for the presence of acellular occluded vessels (integration ocular Olympus $/ 400 \times$ magnification). Each field containing acellular capillary segments was record- ed as positive, and values were normalized to $\mathrm{mm}^{2}$ of retinal area.

The total number of pericytes was counted in ten randomlyselected fields of the retina using an image analysing system (CUE 2; Olympus Opt. Inc., Hamburg, Germany). Differentiation of pericytes from endothelial cells was performed according to the criteria of Kuwabara and Cogan [11]. Pericyte identification was facilitated using a polyclonal anti-vitronectin antibody (rb 69, kindly provided by Klaus Preissner, MaxPlanck-Institut, Bad Nauheim, Germany).

The numbers of pericytes were normalized to the relative capillary density (numbers of cells per $\mathrm{mm}^{2}$ of capillary area).

Additionally, retinae were scored for the presence or absence of microaneurysms.

Electron microscopy. At 56 weeks, three additional animals of each group (NC, DC, D-AG) were anaesthetized by i.p. injection of pentobarbital and whole body perfusion via the left ventricle into the ascending aorta ligated with a knob cannula using $2 \%$ glutardialdehyde in phosphate buffer $(\mathrm{pH}$ $7.2,0.135 \mathrm{~mol}$ ) was performed. Eyes were removed after complete perfusion. Tissues were postfixed in phosphate-buffered $1 \%$ osmium tetroxide, dehydrated through a series of graded ethanol and embedded in Spurr. Semi-thin sections were stained with methylene blue. Ultra-thin sections were mounted on 200 mesh Rb-coated copper grids, stained with uranyl acetate and lead citrate and viewed with a Philips EM 201 transmission electron microscope [12]. Photomicrographs of ten randomly-selected capillaries of the inner nuclear layer were taken at a magnification of $14500 \times$. The width of the retinal capillary basement membrane thickness was measured according to the method of Siperstein, using an image analysing system (Video Plan, Kontron, München, Germany) [13]. Basement membrane proportions in the external circumference of pericytes and between pericytes and endothelial cells were excluded from the examination.

Light and transmission electron microscopic morphometry were performed by two observers blinded to the identity of the samples.

\section{Statistical analysis}

All parameters are given as mean \pm standard deviation. For statistical analysis, analysis of variance (ANOVA) and the Student-Newman-Keuls test was used [14].

\section{Results}

Blood glucose and body weight of treated and untreated rats were essentially identical throughout the study with a slight, but statistically significant, difference between aminoguanidine-treated and untreated diabetic rats at the end of the study. However, Amadori products remained identical and were significantly different from normal controls, as were blood glucose and body weight at each time point (Fig.1). Mean blood glucose of all measurements was $25.73 \pm 1.74 \mathrm{mmol} / \mathrm{l}$ in DC, $23.29 \pm 3.42 \mathrm{mmol} / \mathrm{l}$ in $\mathrm{D}$ $\mathrm{AG}$ and $5.48 \pm 0.41 \mathrm{mmol} / \mathrm{l}$ in $\mathrm{NC}$.

Retinal morphology of non-diabetic rats showed uniform capillary width, an equal distribution of capillary cells and only a few acellular capillaries. The number of pericytes per capillary area and the number of acellular capillaries did not change over time, 

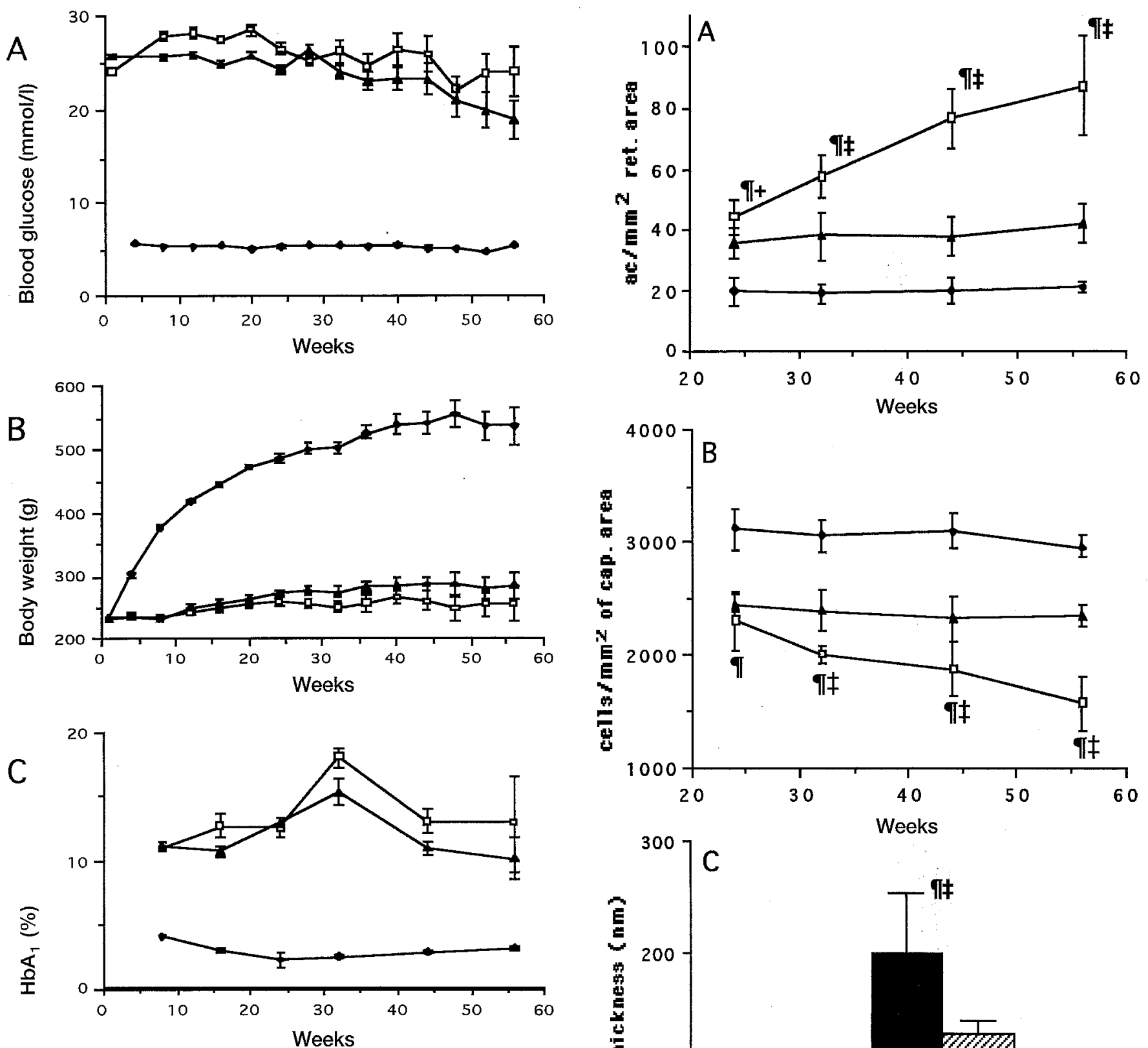

Fig. 1A-C. Blood glucose (A), body weight (B), and $\mathbf{H b A}_{1}(\mathbf{C})$ in non-diabetic $(\mathrm{NC},-$ ), diabetic $(\mathrm{DC},-\square)$ ), and diabetic rats, treated with aminoguanidine (D-AG, $-\mathbf{A}$ ). Results are shown as mean $\pm \mathrm{SD}$

indicating the absence of age-related retinal changes during the period studied. No microaneurysms were observed in the normal group.

In contrast, retinae from untreated diabetic animals progressively developed striking differences in their morphology compared to normal controls. Extended fields with capillary irregularities, cell loss and capillary occlusions occurred.

Quantitatively, after 24 weeks of diabetes, the number of acellular capillaries in DC was $44.6 \pm 5.7$ (NC 24 weeks $19.6 \pm 4.9$ in $\mathrm{NC} ; p<0.001$ ) and increased continuously over time reaching $87.41 \pm 15.1$ after 56 weeks $(p<0.001$ vs DC 24 weeks)(Fig. 2 a).
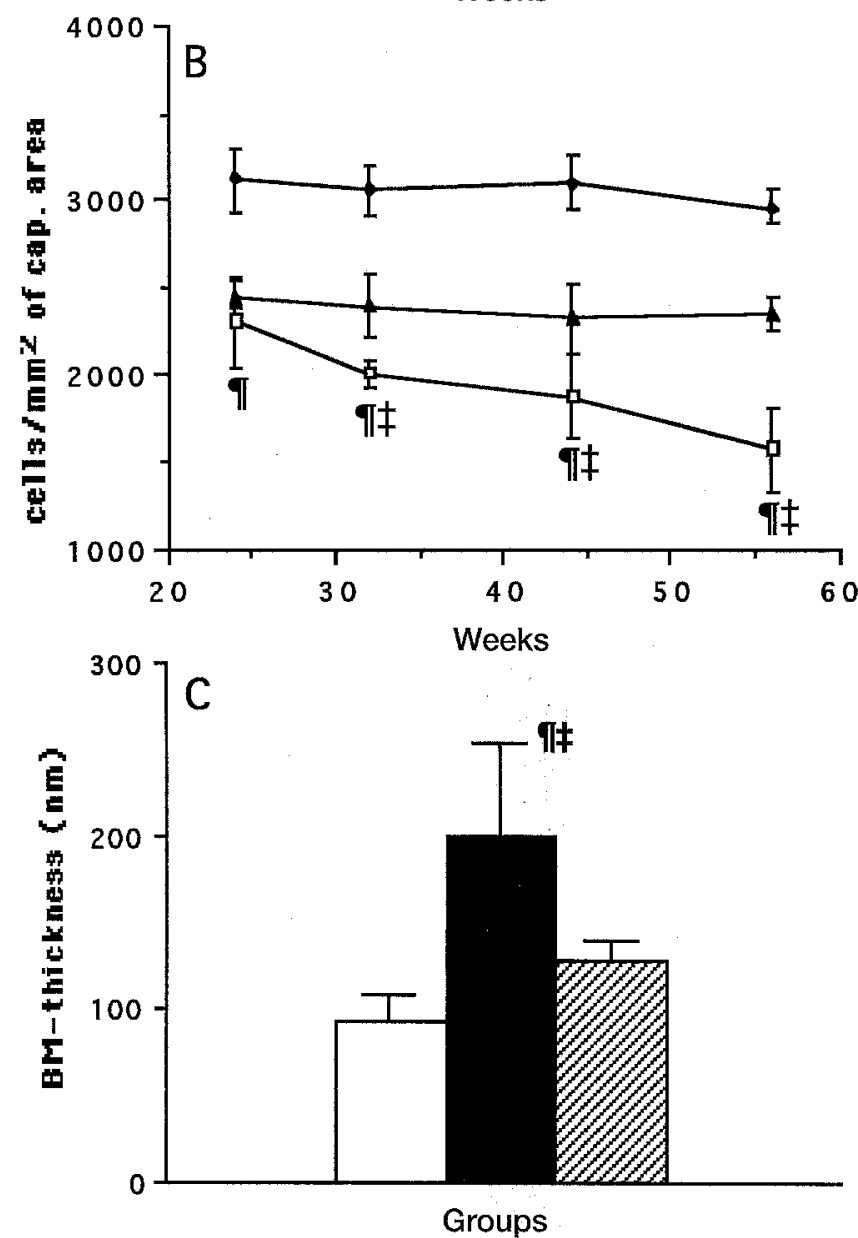

Fig. 2A-C. Time course of acellular capillaries (A) and pericytes $(\mathbf{B})$ in normal $(-)$, diabetic $(-\square)$ and aminoguanidinetreated diabetic rats $(-\mathbf{A})$. Retinal basement membrane thickness (C) measured at the end of the study (56 weeks). $p<0.001$ vs $\mathrm{NC} ;+p<0.05, \ddagger p<0.001$ vs $\mathrm{D}-\mathrm{AG}$

Determined only in areas where capillary occlusions or severe irregularities were absent, chronic hyperglycaemia induced a $47 \%$ pericyte loss from $2310 \pm 170$ after 24 weeks to $1570 \pm 230$ after 56 weeks while NC had $3120 \pm 190$ pericytes $/ \mathrm{mm}^{2}$ after 24 weeks and $2960 \pm 100$ after 56 weeks $(p<0.001$ vs NC for each time point; Fig. 2 b). 
Retinal capillaries of aminoguanidine-treated diabetic animals were strikingly less affected despite exposure to identical levels of chronic hyperglycaemia. Acellular capillaries in D-AG increased over the first 24 weeks to $35.7 \pm 5.18(p<0.01$ vs $\mathrm{NC}$ 24 weeks) and then remained constant for the rest of the time points studied (D-AG 56 weeks $42 \pm 6.20 ; p$ NS vs D-AG 24 weeks, $p<0.001$ vs DC 56 weeks; Fig. 2 a). Aminoguanidine treatment significantly suppressed the progressive pericyte loss after initial diabetic like changes over the first 24 weeks (D-AG 24 weeks $2450 \pm 75 ; p$ NS vs DC 24 weeks; D-AG 56 weeks 2350 $\pm 90 ; \quad p<0,001$ vs DC 56 weeks; Fig. 2 b).

A cumulative number of 14 out of 24 retinae $(58.3 \%)$ of the diabetic group was positive for at least one microaneurysm, the earliest being found after 32 weeks, whereas in aminoguanidine-treated animals only 2 of 25 rats $(8 \%)$ had developed such lesions $(p<0.001)$.

After 56 weeks of diabetes, a significant basement membrane thickening of the retinal capillaries was observed in the diabetic group compared to age-matched non-diabetic rats: $199.8 \pm 55.2 \mathrm{~nm}$ vs $92.9 \pm 15.7$ $\mathrm{nm}(p<0,001)$. Basement membrane thickening was significantly diminished by aminoguanidine treatment (127.8 $\pm 13.0 \mathrm{~nm} ; p<0,001$ vs DC; Fig. $2 \mathrm{c})$.

\section{Discussion}

This study confirms that aminoguanidine prevents the progression of experimental diabetic retinopathy. The stabilizing effect of aminoguanidine on both pericyte loss and the formation of acellular capillaries at a level reached during the initial 24 weeks suggests either a two-component mechanism or a onecomponent mechanism consisting of an early and a late phase. The initial component appears to be noninhibitable by aminoguanidine, whereas the second or late component does.

These time-related characteristics are congruent with essential biochemical features of non-enzymatic glycation [15]. Glucose reacts rapidly with amino groups of proteins forming Amadori products via reversible intermediates (Schiff's base). These early glycation products reach an equilibrium over a period of several weeks $[16,17]$. The percentage of Amadori products as measured by $\mathrm{HbA}_{1}$ in this study was elevated threefold in both diabetic groups irrespective of the treatment compared to the non-diabetic controls. This agrees with the well-known fact that aminoguanidine has no lowering effect on Amadori products [18]. Functional and structural consequences of early glycation on proteins have been extensively studied. Some of these changes have been directly linked to the pathogenesis of diabetic microangiopathy [19].
AGEs are formed from early products in the presence of $\mathrm{O}_{2}$ through complex biochemical rearrangements involving highly reactive free radical intermediates such as 3-deoxyglucosone [20-22]. It is also known that Amadori products catalyse free radical formation which could subsequently cause direct tissue damage by oxidatively modified plasma constituents such as lipids [23].

In one study, aminoguanidine lacking chelating, reducing or antioxidant properties likewise did not affect glycation levels but effectively inhibited glucoxidation product formation, autofluorescence and collagen crosslinking [22]. Therefore, one general mechanism - non-enzymatic glycation - consisting of two components - formation of Amadori products and formation of AGEs - could underly the initiation of vascular changes in diabetic retinae. Aminoguanidine would intervene with the formation of AGEs at a point distal to the Amadori product formation.

The formation of AGEs is a function of time and glucose (Amadori-product) concentration. AGEs irreversibly accumulate on stable macromolecules forming cross-links and sequester proteins that are leaked through permeable vascular walls in diabetes leading to increased vascular wall thickness [24, 25]. Regular degradation mediated by AGE-receptor bearing macrophages is impaired because of reduced susceptibility of AGEs to enzymatic degradation which, in turn, leads to increased cytokine levels that may serve as additional growth-promoting signals $[26,27]$.

Finally, there is evidence from this study that vascular basement membrane thickening which is regarded as the ultrastructural hallmark of diabetic microangiopathy [28] is almost normalized by aminoguanidine treatment from a twofold increase in diabetic rats indicating a predominant role for AGEs in the pathogenesis of basement membrane thickening. This suggests that basement membrane thickening is primarily due to AGE formation.

Taken together, this study suggests that aminoguanidine treatment prevents the progression of experimental diabetic retinopathy, and suggests that non-aminoguanidine-inhibitable mechanisms such as Amadori products and reactive oxygen species are involved in the initial phase of diabetic retinopathy.

Acknowledgements. This study was supported by grant $\mathrm{Ha}$ 1755/1-2 from the German Research Council, Bonn-Bad Godesberg, Germany.

\section{References}

1. Klein R, Klein BEK, Moss S et al. (1984) The Wisconsin Epidemiologic Study of Diabetic Retinopathy. II. Prevalence and risk of diabetic retinopathy when age at diagnosis is less than 30 years. Arch Ophthalmol 102:520-526 
2. Weber B, Burger W, Hartmann R et al.(1986) Risk factors for the development of retinopathy in children and adolescents with type 1 (insulin-dependent) diabetes mellitus. Diabetologia 29:23-29

3. Janka HU, Warram JH, Rand LI et al. (1989) Risk factors for the progression of background retinopathy in longstanding IDDM. Diabetes 38:460-464

4. Chase HP, Jackson WE, Hoops SL et al. (1989) Glucose control and the renal and retinal complications of insulindependent diabetes. JAMA 261:1155-1160

5. Engerman RL, Kern TS (1987) Progression of diabetic retinopathy during good glycemic control. Diabetes 36:808812

6. Constam GR (1977) Sind die diabetische Angio- und Neuropathie vermeidbar? Med Klinik 72:695-702

7. Hanssen KF, Bangstad H-J, Brichmann-Hansen O, DahlJorgensen K (1992) Blood glucose control and diabetic microvascular complications: long-term effects of near-normoglycemia. Diabet Med 9:697-705

8. Brownlee M, Vlassara H, Cerami A (1988) Advanced glycosylation end products in tissue and the biochemical basis of diabetic complications. N Engl J Med 318:1315-1321

9. Hammes HP, Martin S, Federlin K et al. (1991) Aminoguanidine treatment inhibits the development of experimental diabetic retinopathy. Proc Nat Acad Sci USA 88:11555-11558

10. Hammes HP, Brownlee M, Edelstein D et al. (1994) Aminoguanidine inhibits the development of accelerated diabetic retinopathy in the spontaneous hypertensive rat. Diabetologia 37:32-35

11. Kuwabara T, Cogan DG (1960) Studies of retinal vascular patterns. I. Normal architecture. Arch Ophthalmol 64:904-911

12. Syed Ali S (1984) Angioarchitecture of the pancreas of the cat. Light-, scanning- and transmission electron microscopy. Cell Tissue Res 235:675-682

13. Carlson EC, Bjork NJ (1990) SEM and TEM analysis of isolated human retinal microvessel basement membranes in diabetic retinopathy. Anat Rec 226:295-306

14. Sachs L (1992) Angewandte Statistik. 7th edn. Springer, Berlin Heidelberg New York London Paris Tokyo Hong Kong Barcelona Budapest

15. Brownlee M, Vlassara $H$, Cerami A (1988) Advanced glycosylation endproducts and the biochemical basis of complications. Beth Israel Seminar in Medicine. N Engl J Med 318:1315-1321

16. Higgins PJ, Bunn HF (1981) Kinetic analysis of the nonenzymatic glycosylation of haemoglobin. J Biol Chem 256:5204-5208
17. Mortensen HB, Christophersen C (1983) Glycosylation of human haemoglobin $\mathrm{A}$ in red blood cells studied in vitro. Kinetics of the formation and dissociation of haemoglobin $\mathrm{A}_{1 \mathrm{c}}$. Clin Chim Acta 134:317-326

18. Makita Z, Vlassara H, Rayfield E et al. (1992) Hemoglobin-AGE: a circulating marker of advanced glycosylation. Science 258:651-653

19. Brownlee M, Vlassara H, Cerami A (1984) Nonenzymatic glycosylation and the pathogenesis of diabetic complications. Ann Int Med 101:527-537

20. Brownlee M (1990) Advanced products of nonenzymatic glycosylation and the pathogenesis of diabetic complications. In: Rifkin H, Porte D Jr (eds) Diabetes mellitus. Theory and Practice. Alan R Liss, New York, pp 279-291

21. Kato H, Hayase F, Shin DB et al. (1989) 3-Deoxyglucasone, an intermediate product of the Maillard reaction. In: Baynes JW, Monnier VM (eds) Proceedings of the NIH Conference on the Maillard Reaction in Aging, Diabetes and Nutrition. Alan R Liss, New York, pp 69-84

22. Fu MX, Wells-Knecht KJ, Blackledge JA et al. (1994) Glycation, glycoxidation, and cross-linking of collagen by glucose. Kinetics, mechanisms, and inhibition of late stages of the maillard reaction. Diabetes 43:676-683

23. Mullarkey CJ, Edelstein D, Brownlee M (1990) Free radical generation by early glycation products: a mechanism for accelerated atherogenesis in diabetes. Biochem Biophys Res Commun 173:932-939

24. Kent MJC, Light ND, Bailey AJ (1985) Evidence for glucose-mediated covalent cross-linking of collagen after glycosylation in vitro. Biochem J 225:745-752

25. Brownlee M, Vlassara H, Cerami A (1985) Nonenzymatic glycosylation products on collagen covalently trap lowdensity lipoprotein. Diabetes 34:983-941

26. Vlassara H, Brownlee M, Cerami A (1985) High-affinity receptor-mediated uptake and degradation of glucosemodified proteins: a potential mechanism for the removal of senescent macromolecules. Proc Nat Acad Sci USA 82:5588-5592

27. Vlassara H, Brownlee M, Monogue K et al. (1988) Cachectin/TNF and IL-1 induced by glucose-modified proteins: role in normal tissue remodelling. Science 240:1546-1548

28. Rohrbach DH, Martin GR (1982) Structure of basement membrane in normal and diabetic tissue. Ann NY Acad Sci 401:203-211 